\title{
Lipoleiomyoma: An incidental finding in a case of uterine serous adenocarcinoma
}

\author{
John Weichsler, Shane Starr \\ Pathology Department, McLaren Bay Region, Bay City, Michigan, United States
}

Received: May 27, 2020

Accepted: June 16, 20209

Online Published: June 24, 2020

DOI: $10.5430 /$ crcp.v7n1p15

URL: https://doi.org/10.5430/crcp.v7n1p15

\begin{abstract}
We present a rare case of a lipoleiomyoma which was discovered incidentally in a hysterectomy specimen of a 74-year old female patient who underwent surgery for a biopsy proven serous adenocarcinoma of the uterus. Lipoleiomyomas represent uncommon, usually benign soft-tissue tumors, generally found in obese menopausal as well as postmenopausal women aged 50 to 70 years. The reported incidence varies from $0.03 \%$ to $0.2 \%$. The relationship between lipoleiomyomas and gynecologic malignancies has only been reported in a few cases. We report this case of lipoleiomyoma due to its rarity and its association with a serous adenocarcinoma in our patient.
\end{abstract}

Key Words: Lipoleiomyoma, Serous adenocarcinoma, Gynecologic malignancies, Genitourinary pathology

\section{CASE REPORT}

A 74-year-old female reported to our hospital for a scheduled supracervical hysterectomy for the treatment of serous adenocarcinoma of the uterus as diagnosed by biopsy.

The specimen was received in the pathology department in three parts consisting of the right and left pelvic lymph nodes, uterus, bilateral fallopian tubes with ovaries and separate fibroids. Gross examination revealed a specimen measuring $7.5 \mathrm{~cm}$ from fundus to cervical resection margin, $6.0 \mathrm{~cm}$ cornu to cornu and $5.0 \mathrm{~cm}$ from anterior to posterior with a weight of $87 \mathrm{~g}$. The uterine serosa was tan - gray and highly fragmented. Due to its fragmentation, an accurate orientation of the specimen to anterior and posterior was not possible. Ectocervical mucosa was not present. The specimen was bivalved to reveal a highly distorted endometrial cavity measuring $3.0 \mathrm{~cm} \times 2.5 \mathrm{~cm}$, with a nodular, rubbery endometrial layer with a greatest thickness of $0.1 \mathrm{~cm}$. The specimen was sectioned to reveal multiple white - gray, whorled, well circumscribed intramural nodules ranging in size from 0.2 $\mathrm{cm}$ to $4.0 \mathrm{~cm}$ in greatest dimension, the largest of which demonstrated a yellowish component (see Figure 1). A lower uterine segment endometrial lesion was also noted measuring about $1 \mathrm{~cm}$ in greatest dimension and invasive to approximately $0.4 \mathrm{~cm}$, with a total uterine thickness at this point measuring $1.9 \mathrm{~cm}$.

Microscopic evaluation revealed that the lower uterine segment endometrial mass consisted of pleomorphic serous tumor cells arranged in tubular and cribriform structures and focally presented a hobnail appearance (see Figures 2, 3). Mitotic rate was moderately brisk. The tumor superficially invaded the myometrium and lymphatic invasion was not identified. The remaining myometrial masses were leiomyomata, sometimes with hyalinization. Several of these showed frequent admixed adipocytes (see Figure 4, 5).

*Correspondence: John Weichsler; Email: johnweichsler@gmail.com; Address: 2891 Mason Ave., Port Huron, Michigan, 48060, United States. 


\section{DiscuSSION}

Lipoleiomyomas are rare, and usually benign, soft tissue tumors which were first described in 1991 by Meis and Enzinger. ${ }^{[1-3]}$ They are often found in obese menopausal and postmenopausal women between the ages of 50 and 70 years. ${ }^{[1,2,4-6]}$ The reported incidence varies from $0.03 \%$ to $0.2 \% .^{[2,4]}$
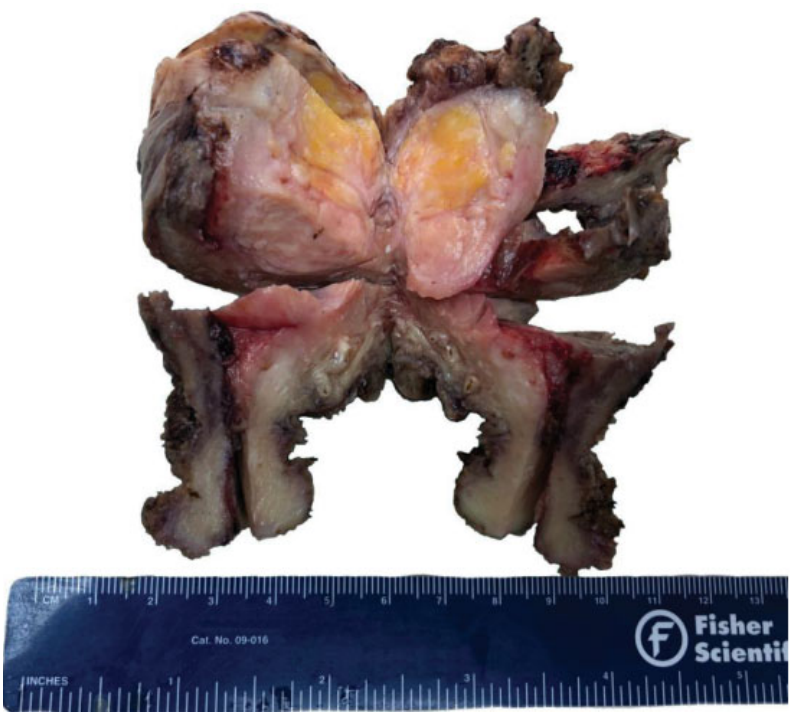

Figure 1. Uterus with attached lower uterine segment demonstrating multiple leiomyoma, the largest of which is a lipoleiomyoma distinguished by its yellow appearance

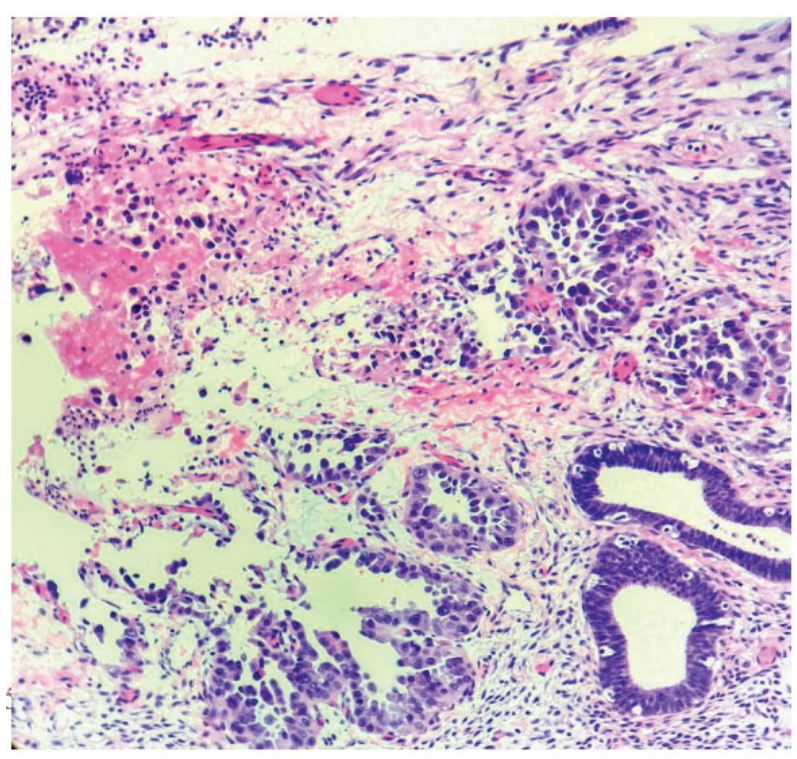

Figure 2. Photomicrograph of the specimen at 100x power demonstrating serous adenocarcinoma with pleomorphic serous tumor cells arranged in tubular and cribriform structures. [Hematoxylin and Eosin Stain]

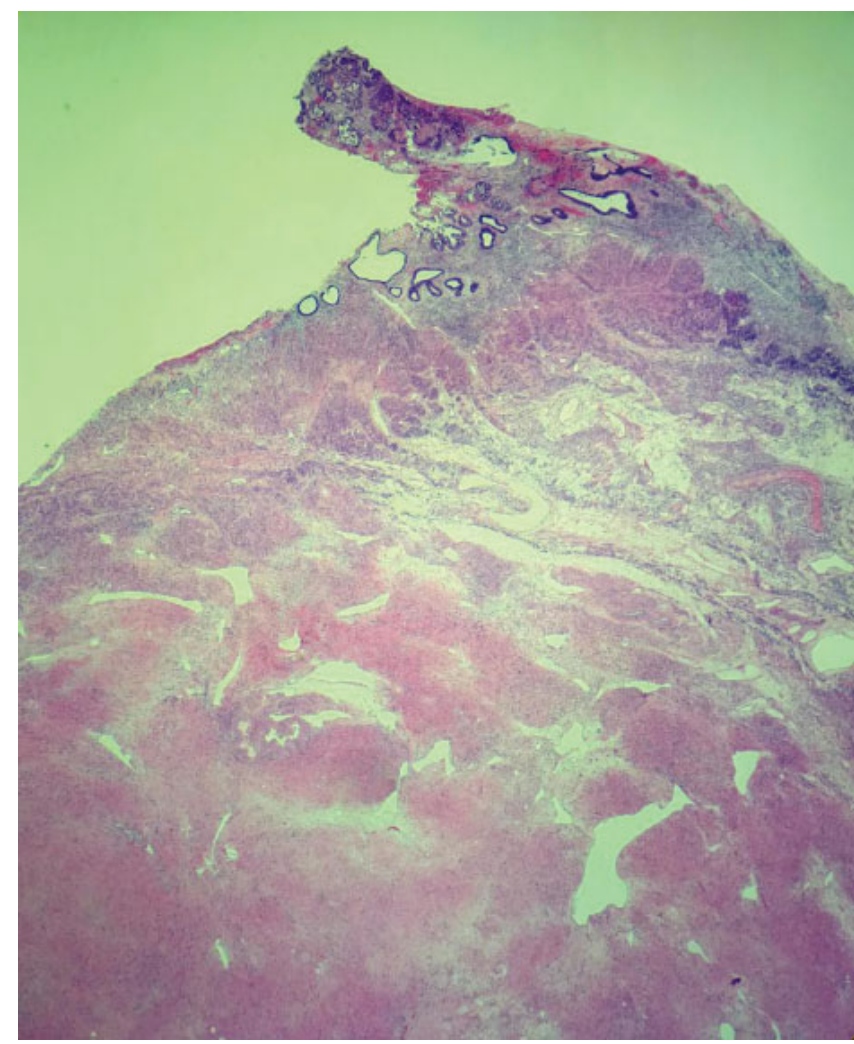

Figure 3. Photomicrograph of the specimen at 20x power demonstrating serous adenocarcinoma with pleomorphic serous tumor cells arranged in tubular and cribriform structures adjacent to the smooth muscle cells of a leiomyoma. [Hematoxylin and Eosin Stain]

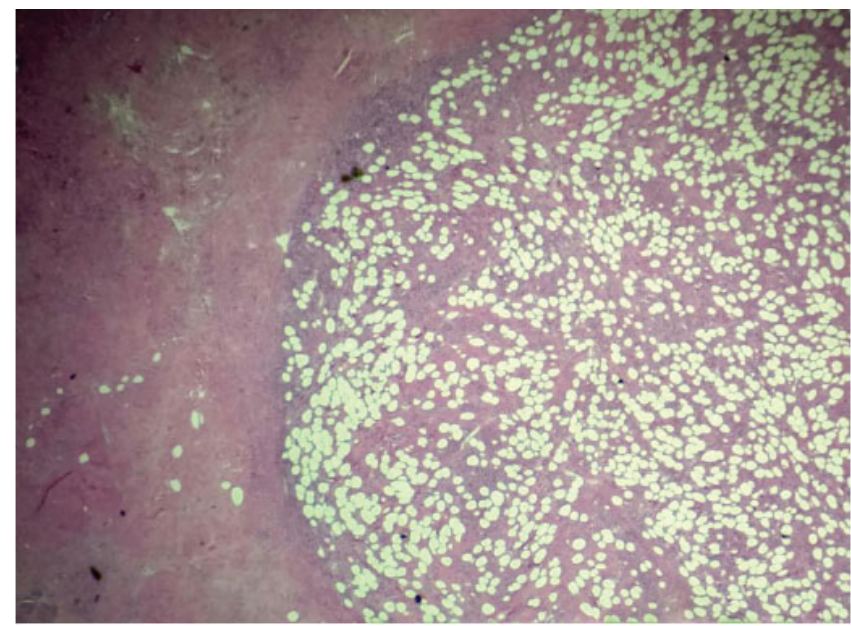

Figure 4. Photomicrograph of the specimen at 20x power showing lipoleiomyoma with an even distribution of adipocytes throughout the lesion. [Hematoxylin and Eosin Stain] 


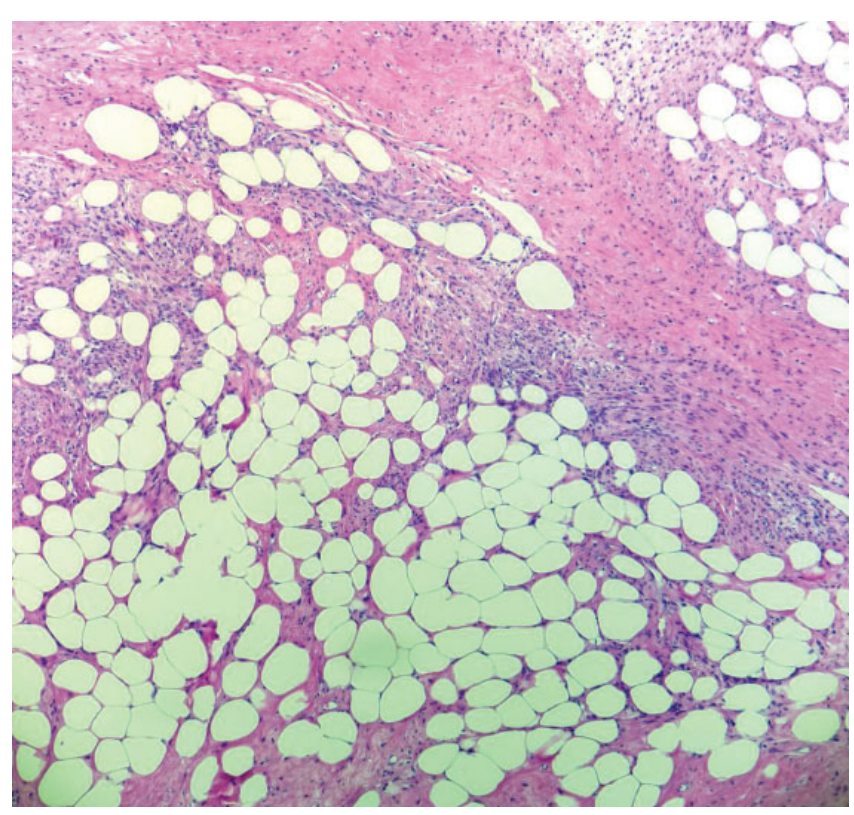

Figure 5. Photomicrograph of the specimen at 100x power showing lipoleiomyoma with an even distribution of adipocytes throughout the lesion. [Hematoxylin and Eosin Stain]

The World Health Organization has two classifications for soft tissue variants of lipomas. Myolipomas of the soft tissue are classified as a variant of lipoma of extrauterine sites thereby distinguishing them as distinct from uterine lipoleiomyoma. ${ }^{[7]}$ Intrauterine lipomatous tumors can be divided into three groups which include pure lipomas (which are composed of encapsulated mature adipocytes), lipomas with various mesodermal components (lipoleiomyomas, angiomyolipomas, and fibromyolipomas), and the rare malignant liposarcoma. ${ }^{[1,2]}$

Most patients with lipoleiomyomas are asymptomatic, however with larger lesions they may present with signs and symptoms like those caused by leiomyomas of similar size. These include a palpable mass, hypermenorrhea and other types of dysfunctional uterine bleeding, pelvic pain, urinary frequency and incontinence. ${ }^{[1,2,4]}$ During patient evaluation, the differential diagnosis includes benign cystic teratoma, malignant degeneration of cystic teratoma, nonteratomatous lipomatous ovarian tumor, benign pelvic lipoma, liposarcoma, lipoblastic lymphadenopathy and pelvic fibromatosis. ${ }^{[1,3,4]}$ Imaging modalities such as ultrasound, computerized tomography $(\mathrm{CT})$, and magnetic resonance imaging (MRI) may be helpful in distinguishing the location of the tumor and possibly elucidate the fatty components; however most diagnoses of lipoleiomyomas are made as incidental findings postoperatively with histopathology ${ }^{[1,6]}$ as with our case. Once a diagnosis of benign leiomyoma or lipoleiomy- oma is established, treatment modalities are very similar. For symptomatic patients, medical management includes analgesics and hormone regulation pills. Surgical options include myomectomy, hysterectomy, myolysis, tumor embolization or radiofrequency ablation. ${ }^{[8,9]}$

For postmenopausal symptomatic patients who are not concerned with fertility, hysterectomy represents a common surgical treatment modality. On gross examination of a surgical specimen, lipoleiomyomas are well circumscribed with a thin connective tissue capsule and are mostly located in an intramural location within the posterior aspect of the uterine corpus. ${ }^{[1]}$ Some lipoleiomyomas are easily distinguished from leiomyomas due to their yellowish coloration.

Traditional microscopic examination begins with the hematoxylin and eosin stain. The histological spectrum for lipomatous tumors ranges from lipoma to fibromyolipoma based upon the variable amounts of smooth muscle, lobules of adipocytes and fibrous tissue contained within the specimen. ${ }^{[1,4]}$ Lipoleiomyomas can be differentiated from leiomyomas with fatty degeneration by the even distribution of adipose tissue throughout the lesion, and from leiomyosarcoma by the bland morphology of the smooth muscle components (see Figure 6). ${ }^{[1,6]}$

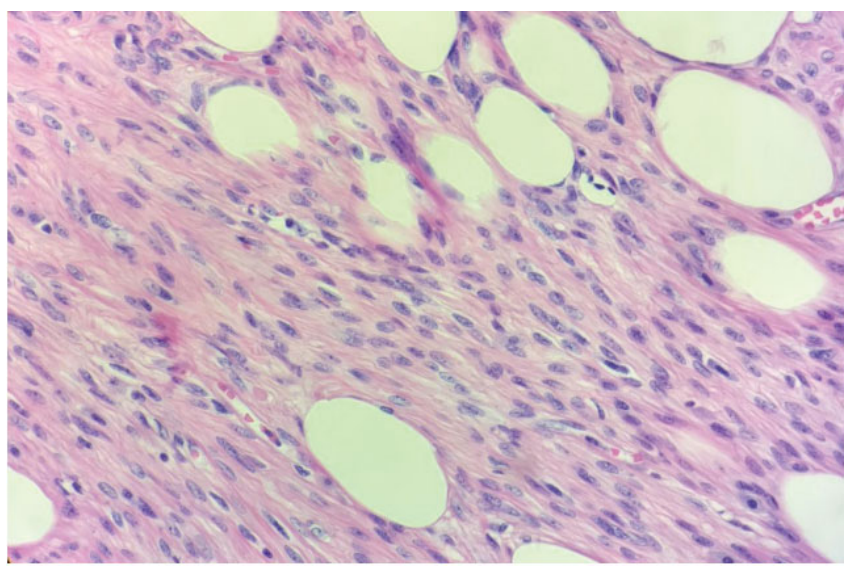

Figure 6. Photomicrograph of the specimen at 200x power showing the bland morphology of the smooth muscle components of the lipoleiomyoma. [Hematoxylin and Eosin Stain]

Previously proposed mechanisms of the pathogenesis included misplaced embryonic fat cells and fatty infiltration or degeneration of connective tissue. ${ }^{[1,2]}$ However, numerous immunohistochemical studies have indicated a complex histogenesis which might arise from multipotent undifferentiated mesenchymal cells or from direct transformation of smooth muscle cells into adipocytes. One study showed focal 
proliferation of adipocytes, labeled by Ki-67 staining, that suggested the adipocytes are neoplastic rather than degenerative. They also showed the presence of estrogen receptors (ER) and progesterone receptors (PR) indicating that the fatty tissue is directly related to the female genital organs. In a study by Akbulut et al., the adipose tissue they studied was positive for Ki-67, desmin, vimentin, and ER and PR receptors, supporting the mechanism of metaplasia of smooth muscle cells into adipose cells. ${ }^{[5,9]}$

The presence of lipoleiomyomas has been commonly associated with metabolic disorders such as hyperlipidemia, hypothyroidism, and diabetes mellitus. ${ }^{[1,9]}$ Many studies have also suggested that a hyperestrogenic state may contribute to their development citing associated lesions such as adenomyosis, endometriosis, endometrial hyperplasia, polyps, complex atypical endometrial hyperplasia and gynecologic carcinomas. $^{[1,5]}$

The relationship between gynecological malignancies which may originate from the uterus, cervix or ovaries and their coexistence with lipoleiomyomas has only been reported in a few cases. In the study by Akbulut et al., ${ }^{[5]} 17 \%$ of the cases examined had an associated gynecological malignancy, including endometrial, ovarian and breast cancers. Gyneco- logical malignancy with estrogen related lesions appeared to be common, presumably related to estrogenic stimulation in these patients. ${ }^{[5]}$ The finding of a lipoleiomyoma in association with serous adenocarcinoma of the uterus in our postmenopausal patient appears to correlate with these studies.

Although further studies on large patient populations are strongly warranted to establish any robust association with malignancy, estrogenic status or metabolic disorder, there is enough evidence to support the fact that once a lipoleiomyoma is discovered, it is important to perform an accurate and detailed clinical and pathological evaluation to search for the presence of a coexisting gynecological neoplasm.

In summary, the authors report a rare case of a lipoleiomyoma which was discovered incidentally in a hysterectomy specimen of a 74-year old female patient who underwent surgery for a biopsy proven serous adenocarcinoma of the uterus. Because of the rarity of lipoleiomyomas, the case report adds interesting information to the field of intrauterine lipomatous tumors.

\section{CONFlicts OF InTEREST Disclosure}

We declare that we have no conflict interests.

\section{REFERENCES}

[1] Nayal B, Somal P, Rao A. Uterine Lipoleiomyoma: A case report of a rare entity. International Journal of Applied Basic Medical Research. 2016 Apr-Jun; 6(2): 134-136. PMid:27127745. https : //doi.org/10.4103/2229-516X.179029

[2] Kumar S, Garg S, Rana P, et al. Lipoleiomyoma of Uterus: Uncommon Incidental Finding. Gynecology \& Obstetrics. 2013; 3: 145 https://doi.org/10.4172/2161-0932.1000145

[3] Manjunatha H, Ramaswamy A, Kumar B, et al. Lipoleiomyoma of uterus in a postmenopausal woman. Journal of Mid-Life Health. 2010 Jul-Dec; 1(2): 86-88. PMid:21716761. https : //doi .org/10.410 3/0976-7800.76219

[4] Bindra R, Sharma N. Uterine Lipoleiomyoma. The Internet Journal of Gynecology and Obstetrics. 2009; 12(2). https ://doi .org/10 $.5580 / 555$

[5] Akbulut M, Gundogan M, Yorukoglu A. Clinical and Pathological Features of Lipoleiomyoma of the Uterine Corpus: A Review of 76
Cases. Balkan Medical Journal. 2014; 31: 224-9. PMid:25625021. https ://doi.org/10.5152/balkanmedj . 2014.13079

[6] Fatnassi R, Ben Ltaifa A, Turki E, et al. Uterine lipoleiomyoma: A case report and literature review. Clincal Obstetrics, Gynecology and Reproductive Medicine. 2017; 3(2): 1-3. https ://doi .org/10.1 5761/COGRM. 1000179

[7] Fukushima M, Schaefer I, Fletcher C. Myolipoma of Soft Tissue: Clinicopathologic Analysis of 34 Cases. American Journal of Surgical Pathology. 2017; 41: 153-160. PMid:27635945. https : //doi.org/10.1097/PAS.0000000000000737

[8] Karaman E, Cim N, Bulut G, et al. A Case of Giant Uterine Lipoleiomyoma Simulating Malignancy. Case Reports in Obstetrics and Gynecology. 2015 Article ID 926961. PMid:26266066. https://doi.org/10.1155/2015/926961

[9] Nazir H, Mehta S, Seena CR, et al. Uterine Lipoleiomyoma: A Report of Two Cases. Journal of Clinical Imaging Science. 2017; 7: 26. PMid:28717557. https://doi.org/10.4103/jcis.JCIS_13_1 7 